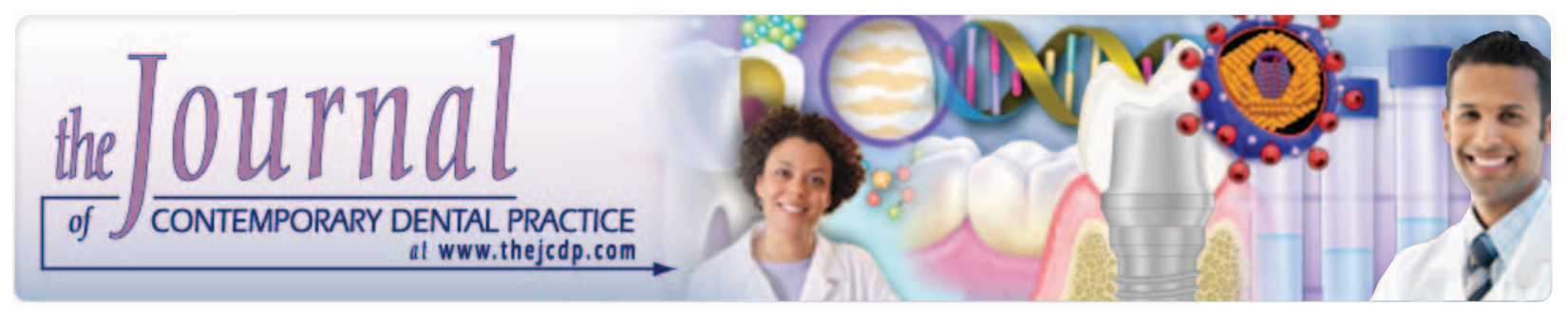

\title{
Effects of Two Antioxidants on the Microleakage of Resin-Based Composite Restorations after Nonvital Bleaching
}

Horieh Moosavi, DDS; Mohammad Javad Moghaddas, DDS, MS; Jamileh Ghoddusi, DDS, MS; Omid Rajabi, PhD

\begin{abstract}
Aim: The aim of this study was to compare the neutralizing effect of antioxidant agents on the microleakage of dental restorations in nonvital teeth after bleaching with carbamide peroxide. Also assessed was the correlation of the microleakage data between an electrochemical and a staining technique.
\end{abstract}

Methods and Materials: Following root canal therapy, 40 sound and similar human maxillary central incisors were randomly divided into four groups of 10 specimens each $(n=10)$. The groups were subjected to one of four treatments: Group 1, bleaching with 10 percent carbamide peroxide for periods of eight hours per day for one week without an antioxidant agent; Group 2, bleaching with 10 percent carbamide peroxide plus 10 percent sodium ascorbate gel; Group 3, bleaching with 10 percent carbamide peroxide plus 10 percent sodium ascorbate gel combined with a surfactant; and Group 4, no bleaching treatment (control group). In all groups, the access cavities were restored using the Single Bond Plus Adhesive system and Z100 resin-based composite. After 1,000 thermal cycles, the teeth were dried and three layers of nail varnish were applied over the entire surface area to within 1.0 $\mathrm{mm}$ of the restoration. A PVC-covered copper wire, with $3.0 \mathrm{~mm}$ of exposed bare wire, was inserted apically $5.0 \mathrm{~mm}$ into the canal, which was obturated with gutta-percha and sealed with sticky wax and varnish at the apex. Leakage was continuously monitored for 40 days using a conductimetric method. The teeth were immersed in 0.5 percent basic fuchsine for 24 hours, followed by sectioning for microscopic examination at 16x magnification. The data were analyzed using ANOVA and the Newman-Keuls multiple comparison, Kruskal-Wallis, Mann-Whitney, and Spearman's rho tests $(p<0.05)$.

Results: Based on the electrochemical results, cavities subjected to 10 percent sodium ascorbate gel combined with a surfactant displayed the least amount of microleakage while teeth that were bleached but not treated with an antioxidant exhibited significantly greater microleakage $(p=0.007)$. These findings also were corroborated by the results of the staining experiments that showed a strong correlation between the electrochemical and staining findings $(p=0.006)$ for the groups.

Conclusion: After nonvital bleaching and the application of the antioxidant 10 percent sodium ascorbate, the addition of the surfactant 0.2 percent Tween 80 to the conventional antioxidant formulation significantly reduced microleakage.

Clinical Significance: The addition of a surfactant to the antioxidant formulation significantly reduced microleakage when it was applied after bleaching.

Keywords: Microleakage, bleaching, carbamide peroxide, 10 percent sodium ascorbate, 0.2 percent Tween 80

Citation: Moosavi H, Moghaddas MJ, Ghoddusi J, Rajabi O. Effect of Two Antioxidants on the Microleakage of Resin-Based Composite 
Restorations after Nonvital Bleaching. J Contemp Dent Pract [Internet]. 2010 December; 11(6):033040. Available from: http://www.thejcdp.com/ journal/view/volume11-issue6-moosavi

\section{Introduction}

Local causes of intrinsic tooth discoloration include pulpal necrosis, intrapulpal hemorrhage, pulp tissue remnants after endodontic treatment, endodontic and coronal filling materials, root resorption, and aging. ${ }^{1}$ It has been reported that 10 percent of endodontically treated teeth are discolored and require bleaching, if only for cosmetic reasons. ${ }^{2}$ A 10 percent preparation of carbamide peroxide has recently been recommended for intracoronal bleaching ${ }^{3-5}$ and was the bleaching agent used in this study. After bleaching, the access cavity should be restored with a bonding agent and resin-based composite to avoid recontamination of the tooth with bacteria and staining substances as well as to stabilize the color of the tooth. ${ }^{1}$

A number of studies have demonstrated that the quality of a resin-tooth bond is poorer immediately after bleaching. $\frac{6-8}{}$ This compromised bonding may be due to a significant decrease in the calcium and phosphate content of the enamel or superficial alterations to the enamel crystallites. $\frac{9.10}{}$ Other potential adverse effects to avoid include an over-etched appearance following acid etching of bleached enamel surfaces, ${ }^{11}$ alterations to the organic matrix in enamel and dentin, 12.13 interference with resin infiltration ${ }^{14,15}$ and polymerization ${ }^{16}$ due to residual oxygen after bleaching, and persistence of the smear layer on dentin surfaces following acid etching. ${ }^{17}$

Several methods have been proposed to eliminate these shortcomings of bleaching, such as removal of the superficial enamel layer, pretreatment of bleached enamel with alcohol, use of adhesives containing organic solvents, cavity cleansing with catalase or 10 percent sodium ascorbate, and a post-bleaching waiting period ranging from 24 hours to three weeks. ${ }^{18}$

In order to avoid the inconvenience and potential discomfort of these strategies, we used an innovative antioxidant material in an effort to improve the adhesion of resin-based composites to nonvital bleached teeth. A second aspect of this study involved an evaluation of the marginal seal of restorations using a dynamic electrochemical method with a comparison of those results to the more common linear dye penetration technique. There is little information on direct comparisons of these test methods.

The null hypotheses of the present study were (1) nonvital bleaching with 10 percent carbamide peroxide has no significant effect on microleakage; (2) the type of antioxidant agent used has no significant effect on microleakage; and (3) the method of microleakage evaluation has no significant effect on the results.

\section{Methods and Materials}

The study employed a total of 40 noncarious human maxillary central incisors that had been freshly extracted due to periodontal disease. The teeth were collected after informed signed consent was obtained from donors under a protocol approved by the Ethics Committee of the University of Mashhad Medical Sciences. The teeth were stored at $4^{\circ} \mathrm{C}$ for up to two weeks in a 0.5 percent chloramine solution. Endodontic access cavities ( 3 $\times 3 \mathrm{~mm}$ ) were prepared using a round diamond bur (No. 4124, KG Sorensen, São Paulo, Brazil) in a high-speed handpiece with air and water cooling. The root canals were instrumented using the step-back technique and filled with sealer (Diaket, 3M/ESPE, Seefeld, Germany) and gutta-percha (Hygienic, Akron, OH, USA) using the cold lateral condensation technique. A $5.0 \mathrm{~mm}$ section at the apical end of the canal was left open to permit insertion of a PVC-insulated copper file $5.0 \mathrm{~mm}$ in length with the terminal $3.0 \mathrm{~mm}$ of wire intentionally left bare. The endodontic filling was removed coronally to a level $2.0 \mathrm{~mm}$ apical to the cementoenamel junction. This point was measured using a periodontal probe in the pulp cavity and reproducing the measurement externally with respect to the CEJ. A 2.0-mm thick glass ionomer base (ChemFil Superior, De Trey Dentsply, Konstanz, Germany) was used as an internal sealing agent during the bleaching process. The 40 teeth were randomly divided into four groups $(n=10)$. In order to simulate clinical conditions, the teeth were mounted with the incisal edges upright in a plastic holder using a high-consistency polysiloxane impression material (Coltene Speedex Putty, Coltene AG, Altstatten, Switzerland). 
Each of the four groups received a different treatment. In Groups 1, 2, and 3 a 10 percent carbamide peroxide preparation (Opalescence, Ultradent Products, South Jordan, UT, USA) was placed into the pulp chamber and access cavity, slightly extending onto the lingual surface. The teeth were bleached for eight hours each day for one week. The specimens were partially immersed in artificial saliva (Dr. Aurafaee Pharmacy Co., Mashhad, Iran) at $37^{\circ} \mathrm{C}$, ensuring that the bleaching gel did not contact the saliva. After completion of the daily bleaching procedure, the pulp chamber and access cavity were irrigated with $2.0 \mathrm{ml}$ of tap water. For the remainder of the day, the specimens were stored in artificial saliva at $37^{\circ} \mathrm{C}$. Prior to immersion in artificial saliva, a cotton pellet was placed into the pulp chamber and the access cavity was sealed with Cavit (3M ESPE, Seefeld, Germany). The artificial saliva was changed twice daily during the one-week period.

\section{Group 1 (bleaching-only group)}

Upon completion of the bleaching procedure, the access cavity and pulp chamber of the specimens in this group were sealed using the Single Bond total-etching dentin adhesive system (3M/ESPE, St. Paul, MN, USA) according to the manufacturer's instructions. After acid etching, two layers of bonding resin were applied with a brush, spread gently with air, and photoactivated using a 20-second exposure to a quartz-tungstenhalogen lamp (Optilux 501, Kerr, Danbury, CT, USA) operating at an irradiance of $450 \mathrm{~mW} / \mathrm{cm}^{2}$. Composite resin (Z100, shade A2, 3M ESPE, St. Paul, MN, USA) was placed and polymerized in $1.5-2.0 \mathrm{~mm}$ thick layers. Each layer was irradiated for 40 seconds.

\section{Group 2 (antioxidant-treated group)}

Immediately after bleaching, the 10 teeth in this group were removed from the plastic holders and the access cavities and pulp chambers were filled with the antioxidant gel 10 percent sodium ascorbate (SA). Each tooth was placed incisal edge down in an individual polystyrene tube (12 $\mathrm{mm} \times 75 \mathrm{~mm}$, Becton, Dickinson and Company, Franklin Lakes, NJ, USA) containing $5 \mathrm{ml}$ of 10 percent SA. The teeth were incubated at $37^{\circ} \mathrm{C}$ for three hours, removed from the tubes, rinsed with an air/water spray, and gently air-dried. The cavities and pulp chambers were restored as previously described for specimens in Group 1.
Group 3 (innovative antioxidant-treated group)

After bleaching, a novel antioxidant agent composed of 10 percent SA gel and a nonionic surfactant $(0.2$ percent Tween 80 , an experimental and noncommerically available material created for this study) was applied in a manner similar to the procedure for Group 2. The teeth were incubated at $37^{\circ} \mathrm{C}$ for three hours. The antioxidant was removed from the teeth by rinsing with an air/water spray and gentle airdrying. Restoration procedures were performed as previously described for specimens in Group 1.

\section{Group 4 (control group)}

Following the root canal procedure, the teeth in this control group were restored in the same way described for specimens in Groups 1, 2, and 3 but without bleaching or antioxidant treatment.

All four groups of teeth were stored in distilled water at $37^{\circ} \mathrm{C}$, and the restorations were finished and polished 24 hours after placement using the Enhance system (LD Caulk/Dentsply, Milford, DE, USA). The specimens were thermocycled from $5^{\circ} \mathrm{C}$ to $55^{\circ} \mathrm{C}$ for 1000 cycles using a one-minute dwell time at each temperature. The teeth were dried and three layers of nail polish (Lâncome, Paris, France) were applied to all surfaces except for a $1.0 \mathrm{~mm}$ zone surrounding the restoration's margins to provide an impermeable barrier to the test fluids. A PVC-insulated copper wire was inserted $5.0 \mathrm{~mm}$ into the root canal so that a $3.0 \mathrm{~mm}$ bare section at the tip of the wire was firmly in contact with the end of the obturation, leaving the remaining $2.0 \mathrm{~mm}$ of the wire covered with PVC insulation. The apical portion of the tooth-wire junction was sealed with sticky wax and three layers of nail varnish. Following wire placement, the teeth were radiographed to ensure correct positioning of the wire. The tooth and inserted copper wire functioned as one electrode in a test circuit, with the other electrode being a strip of stainless steel tape. Both electrodes were immersed in 0.9 percent saline solution. A $20 \mathrm{~V}$ DC potential was applied between the electrodes and current flow in the circuit was detected through the voltage drop across a 1000ohm resistor in series with the voltage source and electrodes. The magnitude of the current indicated the amount of microleakage at the margins of the restorations as well as any percolation through the bulk material. Measurements were obtained for each specimen every other day for 40 days. 
Following the electrochemical leakage study, the wires were removed and the apical portion of the tooth-wire junction was sealed with modeling wax (Dentsply DeTrey, Bois Colombes, France) and three layers of nail varnish. The specimens were immersed incisal edge down in a 0.5 percent basic fuchsine solution for 24 hours at $37^{\circ} \mathrm{C}$. The teeth were removed from the dye solution and washed under tap water, and the nail polish was removed. The teeth were sectioned longitudinally using a low-speed diamond saw (Isomet, Buehler Ltd., Lake Bluff, IL, USA). Sections were made from the buccal to the lingual surface through the center of the coronal restoration and root canal filling material.

\section{Scoring Microleakage}

Microleakage was assessed using a 16x stereomicroscope (Olympus Co., Tokyo, Japan) by two experienced examiners who were unaware of the treatment group assignments. Each examiner judged the depth of the stain and assigned a score independently. If the examiners reported different scores, new readings were performed until a consensus was obtained. Dye penetration was scored using a $0-3$ rating system: $0=$ no leakage, $1=$ dye penetration occurred up to half of the cavity wall depth, $2=$ dye penetration greater than half of the cavity wall depth, and $3=$ dye penetration involving the root canal filling.

\section{Statistical Analyses}

The mean values and standard deviations of the leakage currents obtained using the electrochemical technique were calculated and analyzed using an analysis of variance (ANOVA). A Newman-Keuls multiple comparison test was used to identify any differences between the mean values of the groups. The basic fuchsine staining scores were analyzed by means of a KruskalWallis test performed on the rank order data and pair-wise comparisons were performed using the Mann Whitney-U test. Spearman's rho test was used to correlate the investigators' scores for the staining technique with the results obtained by the electrochemical technique. The level of significance was set at $p<0.05$ for all tests.

\section{Results}

The mean microleakage currents after 40 days for the four test groups are depicted in Figure 1. The curves clearly indicate that the specimens treated with 10 percent SA gel combined with a surfactant displayed the least microleakage, while those bleached and not treated with the 10 percent sodium ascorbate antioxidant displayed a significantly greater amount of microleakage $(p=0.0001)$. Groups treated with 10 percent SA gel and unbleached teeth behaved similarly.

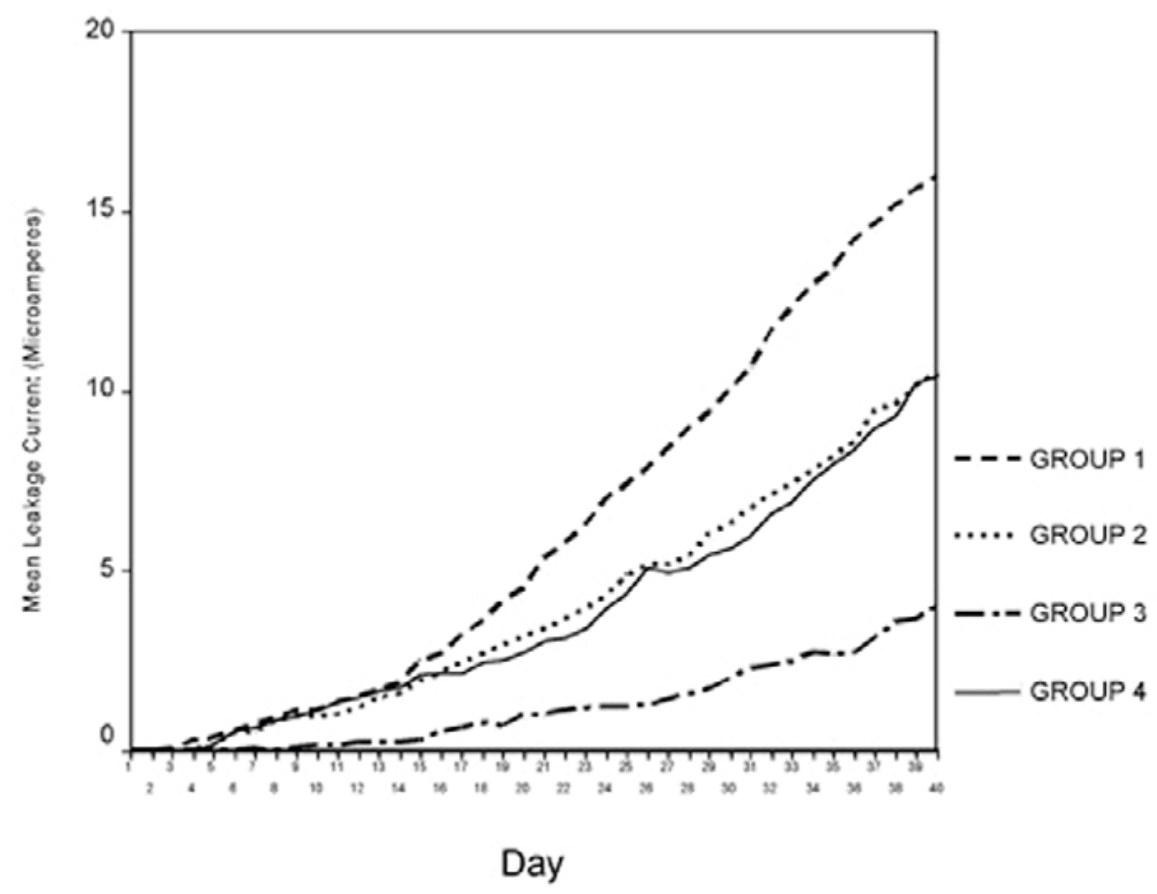

Figure 1. Comparison of electrochemical microleakage test results over 40 days. 


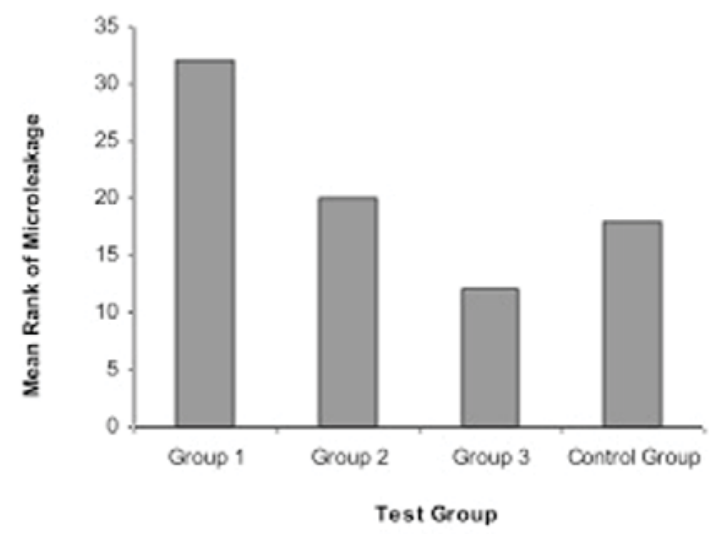

Figure 2. Microleakage mean rank of the test groups.

Table 1. Results of multiple comparison tests between study groups.

\begin{tabular}{|c|c|c|c|c|}
\hline Groups & 1 & 2 & 3 & 4 \\
\hline 1 & & $p=0.012^{\mathrm{a}}$ & $p=0.0001^{\mathrm{a}}$ & $p=0.002^{\mathrm{a}}$ \\
\hline 2 & $p=0.012^{\mathrm{a}}$ & & $p=0.035^{\mathrm{a}}$ & $p=0.653$ \\
\hline 3 & $p=0.0001^{\mathrm{a}}$ & $p=0.035^{\mathrm{a}}$ & & $p=0.049^{\mathrm{a}}$ \\
\hline 4 & $p=0.002^{\mathrm{a}}$ & $p=0.653$ & $p=0.049^{\mathrm{a}}$ & \\
\hline \multicolumn{5}{|l}{${ }^{\mathrm{a}}$ indicates significant statistical differences between groups } \\
\hline
\end{tabular}

Table 2. Spearman's rho results for the correlation between electrochemical and dye penetration microleakage tests.

\begin{tabular}{|c|c|c|c|c|}
\hline $\begin{array}{c}\text { Spearman's } \\
\text { rho results }\end{array}$ & $\begin{array}{c}\text { Group } \\
\mathbf{1}\end{array}$ & $\begin{array}{c}\text { Group } \\
\mathbf{2}\end{array}$ & $\begin{array}{c}\text { Group } \\
\mathbf{3}\end{array}$ & $\begin{array}{c}\text { Group } \\
\mathbf{4}\end{array}$ \\
\hline$R$ & 0.690 & 0.921 & 0.406 & 0.921 \\
\hline$p$-value & 0.025 & 0.0001 & 0.244 & 0.0001 \\
\hline
\end{tabular}

The mean rank values for the staining data are displayed in Figure 2.

The results obtained from multiple comparisons of the study groups are listed in Table 1.

Spearman's rho test was used to establish the correlation between the electrochemical and staining findings $(p<0.05)$ of the groups (Table 2$)$.

Data collected using the electrochemical and the staining techniques were correlated using a Spearman's rho test. A significant correlation was found between the two techniques (Table 2). In Groups 2 and 4, the correlation was $R>0.9$ and statistically significant at $p=0.0001$; for Groups 1 and 3 the correlations were $R=0.690$ and $R=0.406$, and significantly different $(p=0.025$ and $p=0.244$, respectively).

\section{Discussion}

The coronal sealing of teeth following bleaching and antioxidant test treatment was examined using both the electrochemical and linear dye penetration methods. The highest mean leakage values recorded in both test methods were obtained in teeth bleached without antioxidant post-treatment (Group 1), while teeth treated with the innovative 
antioxidant (Group 3) displayed the lowest mean leakage score. These results are consistent with the outcomes of previously reported studies that also attempted to compare different microleakage methods. ${ }^{19-24}$

A perfect restoration with sealed dentin tubules and margins is a prerequisite for successful bleaching therapy.$\stackrel{2,25}{.}$ The appearance of the hybrid layer in bleached enamel is less regular and distinct than in unbleached enamel, which might explain the increased microleakage..$^{26,27}$ In a previous report, ${ }^{28}$ the greatest amount of microleakage was observed in restorations placed immediately after nonvital tooth bleaching, possibly due to delayed release of oxygen that could either interfere with resin infiltration into etched surfaces or inhibit polymerization. $\frac{29}{}$

In the present study, Group 2 and the unbleached controls (Group 4) exhibited similar microleakage behavior. This outcome suggests that the application of an antioxidant may be helpful when restorations are to be placed immediately after bleaching. The lowest microleakage values in both microleakage tests were observed in samples treated with the innovative antioxidant (Group 3). In fact, treatment of the bleached surface in the access cavity with the 10 percent sodium ascorbate gel in combination with 0.2 percent Tween 80, a nonionic surfactant ingredient, may have reduced the surface tension and contact angle as well as the cohesive force between the SA molecules. Consequently the antioxidant agent may actually penetrate the dentin with greater ease, achieving improved efficacy in the dentinal tubules and enamel. In addition, it may be that the free surface energy of the tooth actually increases after treatment due to 0.2 percent Tween 80's detergent effect on the surface, which promotes better sealing. Therefore, the threehour time required for antioxidant treatment may be decreased when the surfactant/antioxidant combination is employed.

Ascorbic acid and its sodium salt are potent antioxidants capable of quenching reactive free radicals in biological systems.$^{30}$ Since ascorbic acid, its salts, and 0.2 percent Tween 80 are nontoxic and widely used in the food industry, it is unlikely that their intraoral use will cause any adverse biological effects or clinical complications. ${ }^{6,7,30}$ Because the efficacy of SA gel was established in a previous study, the gel form was used in this study due to its improved handling characteristics and lower cost compared to the solution formulation. ${ }^{6}$

The experimental conditions of the dye penetration and electrochemical methods are quite different. Dye penetration is a passive method relying on capillary fluid movement, while the electrochemical method is a dynamic process based on the diffusion of ions. ${ }^{31}$ However, some studies employing multiple test methods to compare the sealing ability of restorative techniques used the same samples for each of the tests. ${ }^{21,31}$ There was no discrepancy noted between the results of the staining and electrochemical tests in the experimental groups, although there was the 40-day delay prior to dye penetration assessment when the electrochemical testing was performed.

In the future, it would be advisable to assess the microstructure and bond strength characteristics of teeth treated using our experimental antioxidant formulation to determine if higher microleakage rates observed in vitro translate to significant deterioration of restorations in actual clinical situations. Additionally, the minimum time required for antioxidant treatment after nonvital bleaching should be determined.

\section{Conclusion}

Following the bleaching of nonvital teeth, the addition of the nonionic surfactant 0.2 percent Tween 80 to the conventional 10 percent sodium ascorbate antioxidant significantly reduced microleakage.

\section{Clinical Significance}

The addition of a surfactant such as 0.2 percent Tween 80 to the antioxidant 10 percent sodium ascorbate significantly reduced microleakage when it was applied after bleaching.

\section{References}

1. Plotino G, Buono L, Grande NM, Pameijer $\mathrm{CH}$, Somma F. Nonvital tooth bleaching: a review of the literature and clinical procedures. J Endod. 2008; 34(4):394-407.

2. Baratieri LN, Ritter AV, Monteiro S Jr, Caldeira 
de Andrada MA, Cardoso Vieira LC. Nonvital tooth bleaching: guidelines for the clinician. Quintessence Int. 1995; 26(9):597-608.

3. Leonard RH, Sharma A, Haywood VB. Use of different concentrations of carbamide peroxide for bleaching teeth: an in vitro study. Quintessence Int. 1998; 29(8):503-7.

4. Bizhang M, Heiden A, Blunck U, Zimmer S, Seemann R, Roulet JF. Intracoronal bleaching of discolored non-vital teeth. Oper Dent. 2003; 28(4):334-40.

5. Vachon C, Vanek P, Friedman S. Internal bleaching with $10 \%$ carbamide peroxide in vitro. Pract Periodontics Aesthet Dent. 1998;..10(9):1145-8, 1150, 1152 passim.

6. Kimyai S, Valizadeh $\mathrm{H}$. The effect of hydrogel and solution of sodium ascorbate on bond strength in bleached enamel. Oper Dent. 2006; 31(4):496-9.

7. Lai SC, Mak YF, Cheung GS, Osorio R, Toledano M, Carvalho RM, Tay FR, Pashley $\mathrm{DH}$. Reversal of compromised bonding to oxidized etched dentin. J Dent Res. 2001; 80(10):1919-24.

8. Lai SC, Tay FR, Cheung GS, Mak YF, Carvalho RM, Wei SH, Toledano M, Osorio $\mathrm{R}$, Pashley DH. Reversal of compromised bonding in bleached enamel. J Dent Res. 2002; 81(7):477-81.

9. Perdigão J, Francci C, Swift EJ Jr, Ambrose WW, Lopes M. Ultra-morphological study of the interaction of dental adhesives with carbamide peroxide-bleached enamel. Am J Dent. 1998; 11(6):291-301.

10. Ruse ND, Smith DC, Torneck CD, Titley KC. Preliminary surface analysis of etched, bleached, and normal bovine enamel. J Dent Res. 1990; 69(9):1610-3.

11. Josey AL, Meyers IA, Romaniuk K, Symons AL. The effect of a vital bleaching technique on enamel surface morphology and the bonding of composite resin to enamel. J Oral Rehabil. 1996; 23(4):244-50.

12. Kodaka T, Toko T, Debari K, Hisamitsu $\mathrm{H}$, Ohmori A, Kawata S. Application of the environmental SEM in human dentin bleached with hydrogen peroxide in vitro. $\mathrm{J}$ Electron Microsc (Tokyo). 1992; 41(5):381-6.

13. Hegedüs $C$, Bistey $T$, Flóra-Nagy $E$, Keszthelyi $G$, Jenei $A$. An atomic force microscopy study on the effect of bleaching agents on enamel surface. J Dent. 1999; 27(7):509-15.

14. McGuckin RS, Thurmond BA, Osovitz S.
Enamel shear bond strengths after vital bleaching. Am J Dent. 1992; 5(4):216-22.

15. Torneck CD, Titley KC, Smith DC, Adibfar A. Adhesion of light-cured composite resin to bleached and unbleached bovine dentin. Endod Dent Traumatol. 1990; 6(3):97-103.

16. Rueggeberg FA, Margeson DH. The effect of oxygen inhibition on an unfilled/ filled composite system. J Dent Res. 1990; 69(10):1652-8.

17. Casey LJ, Schindler WG, Murata SM, Burgess JO. The use of dentinal etching with endodontic bleaching procedures. J Endod. 1989; 15(11):535-8.

18. Attin T, Hannig C, Wiegand A, Attin R. Effect of bleaching on restorative materials and restorations-a systematic review. Dent Mater. 2004; 20(9):852-61.

19. Barthel CR, Moshonov J, Shuping G, Orstavik D. Bacterial leakage versus dye leakage in obturated root canals. Int Endod J. 1999; 32(5):370-5.

20. Camps J, Pashley D. Reliability of the dye penetration studies. J Endod. 2003; 29(9):592-4.

21. Delivanis PD, Chapman KA. Comparison and reliability of techniques for measuring leakage and marginal penetration. Oral Surg Oral Med Oral Pathol. 1982; 53(4):410-6.

22. Matloff IR, Jensen JR, Singer L, Tabibi A. A comparison of methods used in root canal sealability studies. Oral Surg Oral Med Oral Pathol. 1982; 53(2):203-8.

23. Pommel L, Jacquot B, Camps J. Lack of correlation among three methods for evaluation of apical leakage. J Endod. 2001; 27(5):347-50.

24. Wu MK, De Gee AJ, Wesselink PR, Moorer WR. Fluid transport and bacterial penetration along root canal fillings. Int Endod J. 1993; 26(4):203-8.

25. Abou-Rass M. Long-term prognosis of intentional endodontics and internal bleaching of tetracycline-stained teeth. Compend Contin Educ Dent. 1998; 19(10):1034-8, 1040-2, 1044 passim.

26. Titley KC, Torneck CD, Smith DC, Chernecky R, Adibfar A. Scanning electron microscopy observations on the penetration and structure of resin tags in bleached and unbleached bovine enamel. J Endod. 1991; 17(2):72-5.

27. Barkhordar RA, Kempler D, Plesh O. Effect of nonvital tooth bleaching on microleakage of resin composite restorations. Quintessence 
Int. 1997; 28(5):341-4.

28. Shinohara MS, Rodrigues JA, Pimenta LA. In vitro microleakage of composite restorations after nonvital bleaching. Quintessence Int. 2001; 32(5):413-7.

29. Kaya $A D$, Türkün M, Arici $M$. Reversal of compromised bonding in bleached enamel using antioxidant gel. Oper Dent. 2008; 33(4):441-7.

30. Inan U, Aydemir H, Tasdemir T. Leakage evaluation of three different root canal obturation techniques using electrochemical evaluation and dye penetration evaluation methods. Aust Endod J. 2007; 33(1):18-22.

31. von Fraunhofer JA, Adachi El, Barnes DM, Romberg E. The effect of tooth preparation on microleakage behavior. Oper Dent. 2000; 25(6):526-33.

\section{About the Authors}

\section{Horieh Moosavi, DDS, MS}

Dr. Moosavi is an assistant professor in the Department of Restorative Dentistry of the School of Dentistry, Dental Material Research Center at Mashhad University of Medical Sciences in Mashhad, Iran. Her research interests include microleakage, adhesive systems and toothcolored restorative materials, stem cells, and tooth regeneration. Dr. Moosavi is a member of the Iranian Academy of Cosmetic Restorative Dentistry.

e-mail: dentist57@yahoo.com
Mohammad Javad Moghaddas, DDS, MS (Corresponding Author)

Dr. Moghaddas is an assistant professor in the Department of Restorative Dentistry and Dental Material Research Center at Mashhad University of Medical Sciences in Mashhad, Iran.

e-mail: moghaddasmj@mums.ac.ir

Jamileh Ghoddusi, DDS, MS

Dr. Ghoddusi is a professor in the Department of Endodontics and the Dental Material Research Center at Mashhad University of Medical Sciences in Mashhad, Iran.

e-mail: ghoddusij@mums.ac.ir

Omid Rajabi, PhD

Dr. Rajabi is an associate professor in the Department of Medicinal Chemistry, Mashad University of Medical Sciences in Mashhad, Iran.

e-mail: rajabio@mums.ac.ir

\section{Acknowledgement}

Funding for this research was provided by the Research Council of Mashhad University of Medical Sciences. The protocol code number for this research study is 86086 and thesis \# 2162 . 